\title{
Ki Hang Kim's Contribution to the Mathematical Social Sciences
}

\author{
Hervé Moulin
}

Published online: 11 April 2013

(C) Springer Science+Business Media Dordrecht 2013

Most pure mathematicians recognize that applied mathematics is an inexhaustible source of new problems and fresh perspectives on old ones. But very rare are those who actually spend a significant part of their intellectual trajectory modeling relevant applied questions with significant mathematical tools.

Ki Hang Kim was one of these precious scholars who greatly contributed to the development of formal models in the social sciences, by regularly crossing the bridge to economics, psychology, or political sciences, and contributing remarkable papers in those disciplines. He published on consensus theory in the Journal of Mathematical Psychology, then in the Journal of Economic Theory on preference aggregation and decision theory. I saw his name first in his work on voting theory, where he discovered several new restricted domains of preferences with non dictatorial voting rules (Math. Social Sci. 1980). He even contributed to pure game theory (Math. Social Sci. 1982, and 1984) and its applications to industrial competition (Math. Social Sci. 1988).

In the community of mathematical social scientists, Ki Hang Kim will be remembered mostly for the launching in 1980 of a new journal where all mathematical techniques used throughout social sciences would reflect on one another, and where the work of pure mathematicians with an applied flavor would be welcome. The birth of Mathematical Social Sciences was very timely, just about when the social sciences disciplines started to embrace Game Theory and Social Choice Theory. I had the privilege to succeed Ki Hang at the helm of the journal in 1993, and do my part to make of his brainchild a high quality scholarly outlet with a distinct personality, and a very bright future.

H. Moulin (凶)

Department of Economics, Rice University, Houston, TX 77251-1892, USA

e-mail:moulin@rice.umd.edu 